Ann. Biol. anim. Bioch. Biophys., 1979, 19 (6), 1807-1816.

\title{
Etude du matériel sécrété dans la chambre de régénération d'un appendice locomoteur de Porcellio dilatatus Br. (Crustacea : Isopoda)
}

\author{
par G. NOULIN
}

avec la collaboration technique de Colette BESSE, B. LEBOURG, J. C. ARTAULT et Th. BAUVAIS

\author{
Laboratoire de Physiologie ef Génétique des Crustacés \\ (E. R. A. C. N. R. S. no 230). \\ Service Général de Microscopie Electronique Appliquée d̀ la Biologie. \\ 40, Avenue du Recteur Pineau 86022, Poifiers Cedex.
}

Summary. Material secreted in the incubation chamber of a regenerating bud of Porcellio dilatatus Br. (Crustacea : Isopoda).

A regenerating appendage of $P$. dilatatus develops in an incubation chamber located in the stump. This chamber is filled with a fibrous, alveolar material containing chitin and an acid, non-sulphated mucosubstance hydrolyzed by testis hyaluronidase. The apical surfaces of the epithelial cells of the regenerating bud show differentiations - very short, dark-tipped microvilli and carrier vesicles - which are probably involved in the secretion of the material.

L'étude histologique de la régénération d'un appendice d'isopode montre que le bourgeon, mis en place après cicatrisation, se développe dans une chambre délimitée : 1) par le caillot hémostatique formé par des hémocytes au moment de l'ablation, 2) par l'exosquelette du moignon, 3) par l'épithélium cicatriciel rétracté (Needham, 1965 ; Hoarau, 1973 ; Noulin et Maissiat, 1974). Cette chambre est, dès sa formation, remplie par un matériel fibreux et alvéolaire qui, après les colorations topographiques utilisant le bleu d'aniline ou le vert lumière, a les mêmes affinités tinctoriales que l'exosquelette. Ce mode de régénération est différent de celui des crustacés décapodes chez lesquels le régénérat se développe extérieurement, protégé par un sac membranaire qui le moule étroitement en restant mince et flexible (Busson, 1935 ; Nouvel-Van Rysselberge, 1937 ; Bauchau, 1961). Ce sac membranaire des décapodes forme une cuticule dont la sécrétion par l'épithélium cicatriciel commence sous la croûte d'hémocytes (Adiyodi, 1972 ; Bazin et Demeusy, 1972).

Le matériel fibreux observé chez les isopodes est comparable, par sa position et son aspect, à la couche gélatineuse que Marks et Reinecke (1964) ont observée autour du régénérat, cultivé in vitro, de la patte de la blatte Leucophea maderae, et que Marks 
ef Leopold (1971) considèrent comme un complexe protéine-glucide. Nous avons recherché, à l'aide de quelques tests histochimiques si le contenu de la chambre d'incubation du régénérat d'un oniscoïde renferme également des mucosubstances. Cetle étude histochimique a été complétée par une étude cytologique pour préciser la structure ef l'origine de ce contenu.

\section{Matériel et méthodes.}

Les animaux utilisés sont des Porcellio dilatatus mâles à la période $C$ du cycle de mue, provenant d'un élevage du laboratoire. Après anesthésie au dioxyde de carbone, nous avons sectionné les appendices locomoteurs de la sixième paire à la base du méropodite, juste au-dessus de l'articulation avec l'ischiopodite. Les animaux ont été maintenus à la température de $20^{\circ} \mathrm{C}$ jusqu'à leur sacrifice.

Les tests histochimiques ont été effectués sur des régénérats d'animaux fixés de 5 à 21 jours après l'ablation ; 5 jours après l'ablation, l'épithélium a rétabli sa continuité sous le caillot hémostatique ; 21 jours après l'ablation, le régénérat est replié dans ta chambre et présente des constrictions nettes limitant les articles reconstitués. Le contrôle du stade du cycle de mue a été fait au moment de la fixation : tous les animaux ont été sacrifiés alors qu'ils étaient encore à la période $C$. Les liquides fixateurs que nous avons employés sont les liquides de Bouin, de Carnoy, de Duboscq-Brasil ef le formol. Les tests histochimiques utilisés sont :

- l'acide periodique-Schiff (APS) qui met en évidence des groupements vic-glycol ; - la fuchsine paraldéhyde sans oxydation préalable qui signale la présence de groupements électronégatifs ;

- le bleu alcian (8GS) à pH 2,5 qui colore les mucosubstances acides (MPSA) et les sialomucines ;

- la métachromasie du bleu de toluidine ou de l'azur $\mathrm{A}$ à $\mathrm{pH} 1,4, \mathrm{pH} \mathrm{3,4}$ ou pH 4,2 ; une métachromasie apparaissant à des $\mathrm{pH}$ supérieurs à 3,5 décèle la présence de groupements acides, carboxyle et/ou sulfate ; une métachromasie apparaissant à des $\mathrm{pH}$ inférieurs à 3,5 est due à la présence de groupements sulfate ;

- le bleu alcian à pH 0,5 (colore les MPSA sulfatés) associé au jaune alcian à pH 2,5 (colore les MPSA carboxylés) ;

- le bleu alcian à pH 2,5 associé à l'APS ;

- la méthylation, qui bloque tous les groupements acides des MPSA, suivie ou non de saponification qui élimine les groupements sulfatés, mais qui rétablit l'électronégativité des groupements carboxyle ;

- la digestion à la hyaluronidase d'origine testiculaire bovine (Sigma type I S) employée soit à $\mathrm{pH} 6$ pendant $5 \mathrm{~h}$ à $37^{\circ} \mathrm{C}$, soit à $\mathrm{pH} 4,4$ pendant $5 \mathrm{~h}$ à $37^{\circ} \mathrm{C}$ dans $\mathrm{CINa}$ $0,1 \mathrm{M}$ ef suivie de la coloration au bleu alcian ( $\mathrm{pH} 2,5)$-APS. La hyaluronidase testiculaire hydrolyse l'acide hyaluronique, la chondroitine et les chondroïtine-sulfates A et $\mathrm{C}$;

- l'hydrolyse acide à $\mathrm{pH} 2,5$ (tampon acétate) pendant 2 ou $3 \mathrm{~h}$ ou à pH 1,4 $\left(\mathrm{SO}_{4} \mathrm{H}_{2}\right)$ pendant $2 \mathrm{~h}$ à $60^{\circ} \mathrm{C}$ avant coloration par l'APS ou par le bleu alcian-APS ; l'hydrolyse acide élimine les groupes acide sialique; 
- la digestion à la chitinase extraite de Streptomyces griseus (Calbiochem, $n^{\circ} 220471$ ou Sigma, $\mathrm{C}$ 6137) utilisée à $\mathrm{pH} 6$ à $35^{\circ} \mathrm{C}$ pendant 24 h avant coloration à l'APS.

L'étude cytologique a été faite sur des régénérats de 9 à 11 jours. Ils ont été fixés, soit au glutaraldéhyde seul (glutaraldéhyde à 3 p. 100 dans du tampon phosphate ou cacodylate $0,1 \mathrm{M}$ ), soił au glutaraldéhyde puis à l'acide osmique à 2 p. 100 dans le même tampon. Ils ont été inclus dans l'araldite après déshydratation à l'acétone. L'étude ultrastructurale morphologique a été faite après contraste sur coupes par l'acétate d'uranyle et le citrate de plomb. Cette étude a été complétée par la détection cytochimique des saccharides par la méthode à l'acide periodique-thiocarbohydrazide-protéinate d'argent (AP-TCH-ProtAg) (Thiéry, 1967 ; Thiéry et Rambourg, 1974).

\section{Résultats.}

La réaction à l'APS est faiblement positive sur le contenu de la chambre d'incubation du régénérat à tous les moments de son développement que nous avons observés.

Au début du développement du régénérat, tout le contenu de la chambre est coloré par le bleu alcian et, de façon nette mais peu intense, par la fuchsine paraldéhyde. A partir du 9e ou 10e jour, ces deux colorations permettent de distinguer ce que nous appellerons «l'enveloppe du régénérat» qui ne se colore ni par le bleu alcian, ni par la fuchsine paraldéhyde, mais prend les colorants de fond. Cette enveloppe forme un manchon de quelques $\mu \mathrm{m}$ d'épaisseur autour de l'extrémité apicale du régénérat. Elle n'est pas étroitement accolée à la surface du régénérat, mais, plus ou moins plissée, en est éloignée de $20 \mu \mathrm{m}$ au plus (fig. 3). Après la coloration combinée bleu alcian-APS, l'enveloppe est APS-positive alors que le reste du contenu est bleu furquoise.

Le bleu de toluidine ou l'azur A, utilisés à pH 4,20 donnent au contenu de la chambre une coloration violette (métachromasie $\beta$ ) qui n'est conservée que si les coupes ne sont pas lavées à l'eau mais essorées et déshydratées à l'alcool butylique tertiaire. Utilisés à $\mathrm{pH} 1,4$ ou $\mathrm{pH} 3,4$, ils ne colorent pas ce contenu. Cette métachromasie faible est l'indice de la présence de fonctions carboxyle. La coloration combinée bleu alcian-jaune alcian et les réactions de méthylation ef de méthylation-saponification (voir résultats dans tabl. 1) confirment la présence de groupements carboxyliques et ne décèlent pas celle de groupements sulfatés dans le contenu de la chambre d'incubation.

L'action des enzymes ou des hydrolyses acides a donné les résultats suivants :

- la hyaluronidase à pH 6 ne modifie pas la colorabilité du contenu de la chambre par le bleu alcian. Par contre, lorsque la hyaluronidase est utilisée à pH 4,4, la coloration n'est plus bleu furquoise mais violacée eł peu intense ;

- les hydrolyses acides à $\mathrm{pH} 2,5$ et 1,4 ne changent pas les réactions du contenu de la chambre aux colorations par l'APS ou le bleu alcian-APS ;

- l'action de la chifinase détruit en partie le matériel fibreux qui entoure le régénérat ef supprime la colorabilité par l'APS.

L'observation de l'ultrastructure du contenu de la chambre d'incubation montre qu'il est formé de deux types d'éléments ; des filaments fins mêlés à des fibres épaisses 
TABLEAU 1

Réactions du contenu de la chambre d'incubation ef de l'enveloppe du régénérat aux tests histochimiques et aux digestions enzymatiques

\begin{tabular}{|c|c|c|}
\hline Tests & $\begin{array}{l}\text { Contenu de la chambre } \\
\text { du régénérat }\end{array}$ & Enveloppe du régénérat \\
\hline$\cdots \cdots$ & $\dashv-$ & + \\
\hline Fuchsine paraldéhyde sans oxydation ....... & $t+t$ & 0 \\
\hline $\begin{array}{l}\text { Bleu acian }(\mathrm{pH} 2,45) \\
\text { Bleu alcian après méthylation } \ldots \ldots \ldots \ldots \ldots \ldots \\
\text { Bleu alcian après méthylation et saponifica- } \\
\text { tion } \ldots \ldots \ldots \ldots \ldots \ldots \ldots \ldots \ldots \ldots \ldots \ldots\end{array}$ & $\begin{array}{c}+++ \\
0 \\
++++\end{array}$ & $\begin{array}{r}0 \\
0 \\
+++\end{array}$ \\
\hline 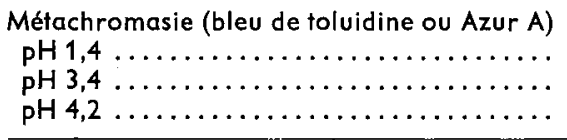 & $\begin{array}{c}0 \\
0 \\
\text { métachromasie } \beta\end{array}$ & $\begin{array}{l}0 \\
0 \\
0\end{array}$ \\
\hline 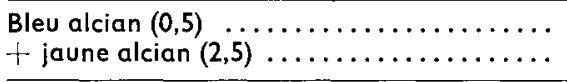 & $\begin{array}{r}0 \\
+++ \\
\end{array}$ & $\begin{array}{l}0 \\
0\end{array}$ \\
\hline 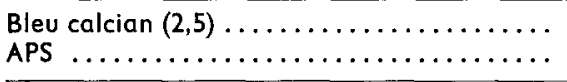 & $+t_{0}^{+-}$ & $\begin{array}{c}0 \\
+\end{array}$ \\
\hline 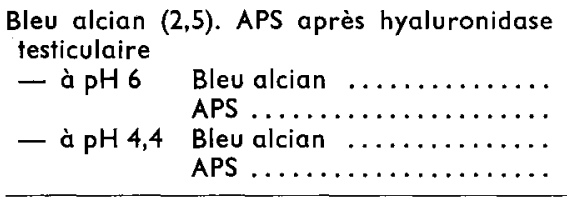 & $\begin{array}{c}+++ \\
0 \\
+- \\
+-\end{array}$ & $\begin{array}{l}0 \\
+ \\
-\end{array}$ \\
\hline 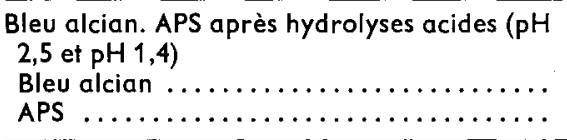 & 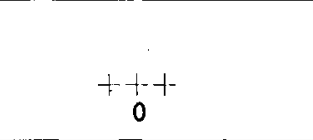 & $\begin{array}{l}0 \\
+\end{array}$ \\
\hline APS après chitinase $\ldots \ldots \ldots \ldots \ldots \ldots \ldots \ldots$ & 0 & - \\
\hline
\end{tabular}

0 pas de réaction, $\nmid-$ réaction faible, $+\nmid-$ réaction moyenne, $+-\nmid+$ réaction forte.

\section{PLANCHE I.}

FIG. 1. - Montage in toto d'un régénérat; moignon prélevé 7 jours après l'ablation.

FIG. 2 et 3. - Coupes histologiques du moignon ; fig. 2. - Prélevé 5 jours après l'ablation ; fig. 3. prélevé 14 jours après l'ablation.

Le trait sur les figures 1,2 et 3 représente $100 \mu \mathrm{m} ; \mathrm{c}$ : bouchon hémostatique ; $c$. i. : chambre d'incubation du régénérat; e : enveloppe du régénérat; ep. $c$. : épithélium cicatriciel ; $r$ : bourgeon de régénération. 

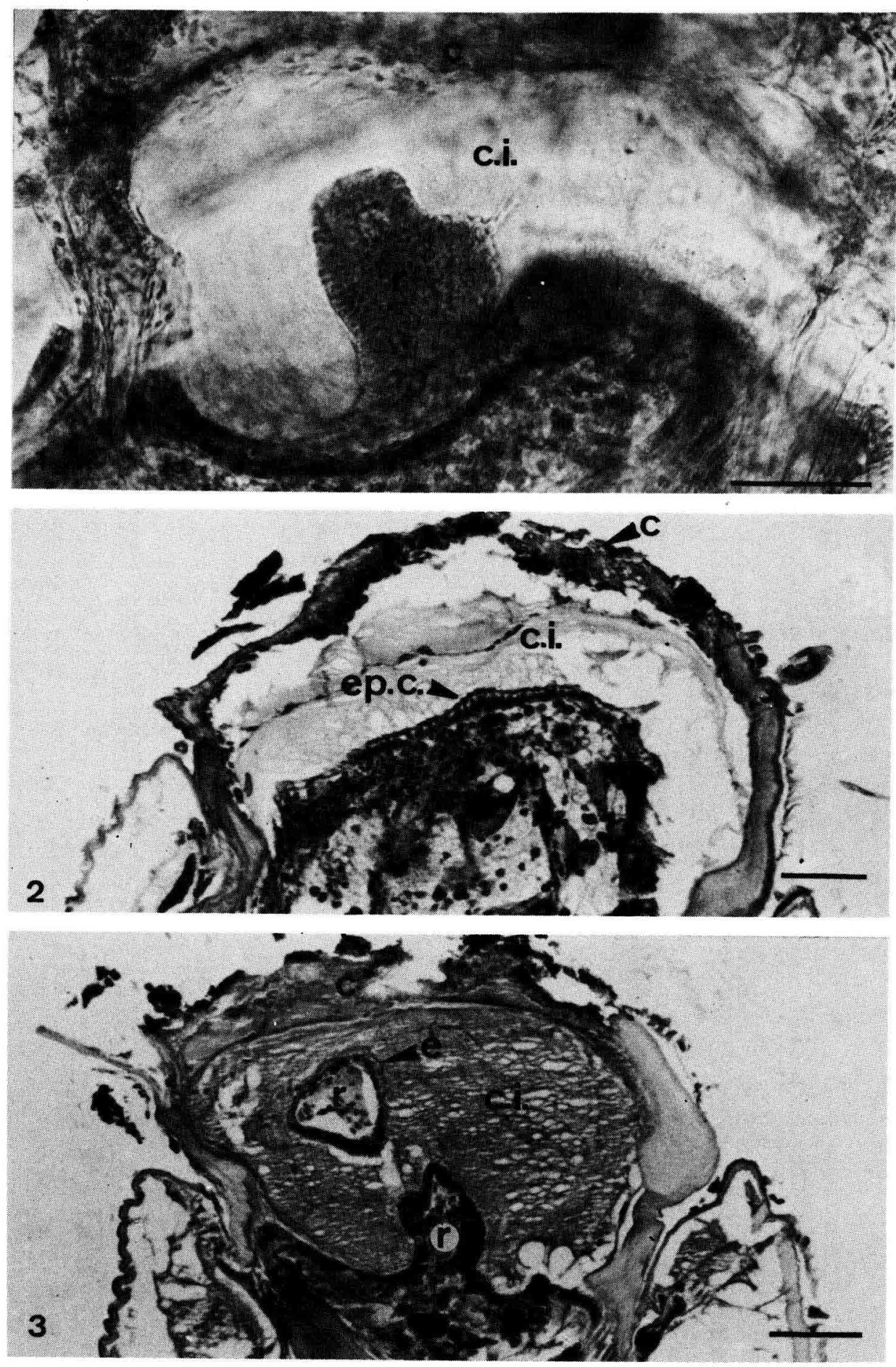
et sinueuses de matériel granuleux (fig. 4). L'enveloppe du régénérat, présente seulement chez les animaux fixés 11 jours après l'ablation, a un aspect granuleux comparable aux fibres épaisses du contenu de la chambre. Cette enveloppe forme une couche continue, décollée et plus ou moins éloignée du pôle apical des cellules épithéliales (fig. 5 et 6 ).

Les cellules épithéliales du bourgeon de régénération présentent des différenciations au niveau de leur membrane plasmique. II n'est pas rare d'observer des vésicules ouvertes à leur pôle apical. Une substance dense aux électrons semble être libérée par ces vésicules (fig. 5). De plus, la membrane apicale de ces cellules porte, par endroits, quelques microvillosités très courtes et dont les sommets sont épaissis. Très souvent, les sommets de ces microvillosités sonf reliés entre eux par un trait de matériel dense aux électrons (fig. 6).

La réaction au protéinate d'argent marque nettement les filaments minces du contenu de la chambre sur les coupes traitées pendant 48 ou $72 \mathrm{~h}$ à la TCH. Les travées de matériel granuleux semblent également se colorer à l'AP-TCH-ProtAg ; mais nos observations ne permettent pas de déterminer si ce marquage est dû à la réaction propre du matériel de ces travées ou à des filaments fins qui s'y accolent (fig. 7 ).

\section{Discussion.}

a) Nature chimique du confenu de la chambre du régénérat.

Le matériel fibreux de la chambre d'incubation du régénérat réagit nettement aux colorations qui mettent en évidence les groupements carboxyle, mais pas à celles des groupements sulfate. Ce matériel peut donc contenir soit des mucosubstances à groupements carboxyliques, soit des sialomucines. Les hydrolyses acides ne modifiant pas la colorabilité par l'APS ou par le bleu alcian, il semble donc que le contenu de la chambre ne contient pas d'acide sialique. Au contraire, la hyaluronidase testiculaire supprime en partie la colorabilité du matériel fibreux par le bleu alcian. Certes, la

\section{PLANCHE II.}

FIG. 4. - Contenu de la chambre d'incubotion composé de filaments fins et de fibres épaisses et sinueuses ; moignon prélevé 9 jours après l'ablation.

FIG. 5. - Vésicule d'exocytose du pôle apical d'une cellule épithéliale, sous l'enveloppe du régénérat (e) ; moignon prélevé 11 jours après l'ablation.

FIG. 6. - Alignement de microvillosités au pôle apical d'une cellule épithéliale. Un filament est tendu entre les sommets épaissis des microvillosités (pointe de flèche); moignon prélevé 11 jours après l'ablation.

FIG. 7. - Contenu de la chambre après réaction à l'acide periodique-thiocarbohydrazide-protéinate d'argent. Les filaments fins sont marqués (pointes de flèche) ; moignon prélevé 9 jours après l'ablation. e : enveloppe du régénérat. 
hyaluronidase n'a pas une spécificité d'action très étroite et peut hydrolyser l'acide hyaluronique, la chondroïtine et des chondroïtine-sulfates, mais la comparaison de son action avec les résultats des autres tests utilisés limite l'interprétation de son action à l'hydrolyse de l'acide hyaluronique, ou de la chondroïtine. La forme non sulfatée de la chondroïtine étant peu répandue dans le règne animal (Hunt, 1970), s'il s'agit bien de l'un de ces deux mucopolysaccharides acides (MPSA) il est plus probable que ce soit l'acide hyaluronique.

Lorsque l'APS est utilisé seul, il colore faiblement le contenu de la chambre. Cette réaction est confirmée en cytochimie ultrastructurale par l'AP-TCH-ProtAg dont la spécificité recouvre celle de l'APS (Thiéry et Rambourg, 1974). Il y aurait donc une autre substance en plus de l'acide hyaluronique qui n'est pas APS-positif (Pearse, 1968 ; Ganter et Jolles, 1969). Or, la réaction à l'APS disparaît après hydrolyse par la chitinase. La réactivité de la chitine à l'APS est controversée, cependant, la chitine nouvellement formée et non sclérifiée peut être APS-positive (Lison, 1960 ; Ganter et Jolles, 1969), et c'est probablement sous cette forme « jeune » que la chitine peut être présente dans le contenu de la chambre.

Chez les invertébrés, quelques études ont été faites sur les mucopolysaccharides (MPS) et les glycoprotéines des tissus en voie de cicatrisation ou de régénération. Nos résultats rejoignent ceux de Sannasi (1968) qui signale la présence d'acide hyaluronique dans le tissu cicatriciel formé après élimination d'une petite surface de l'épiderme de Periplanefa americana. Marks et Reinecke (1971) n'ont pas trouvé de chitine dans la couche gélatineuse située autour du régénérat de la patte de Leucophea maderae.

Chapron $(1972,1973)$ met en évidence des glycoprotéines et des MPS détruits par la hyaluronidase dans le tissu cicatriciel d'Eisenia foetida. Chez ce ver, ces substances, dont l'apparition accompagne les remaniements des surfaces cellulaires, interviennent dans la mise en place du blastème de régénération. Il ne semble pas que ce rôle puisse être aftribué aux MPSA que nous trouvons autour du régénérat de Porcellio dilatatus, d'une part, parce qu'ils sont situés à l'extérieur de l'épithélium qui délimite ce régénérat, d'autre part, parce que leur production est plus abondante pendant la croissance de ce dernier qu'au moment de sa formation. Par sa position, le contenu de la chambre du régénérat a certainement un rôle protecteur, en maintenant autour du bourgeon un milieu favorable à son développement. En effet, $P$. dilałałus est un crustacé terrestre, et le caillot hémostatique seul peut être insuffisant pour protéger le bourgeon de régénération contre la dessiccation (ou contre des infections).

b) Origine du matériel fibreux de la chambre du régénérat.

Avant la formation du bourgeon et pendant les premières heures de son développement, des hémocytes sont présents dans la chambre d'incubation, dont le volume est alors faible. Chez les Crustacés décapodes, les hémocytes contiennent et peuvent libérer des protéines et des glycoprotéines lors des processus de coagulation (Wood et al., 1971 ; Bauchau et de Brouwer, 1974 ; Bauchau et al., 1975 ; Durliat ef Vranckx, 1976 ; Bauchau et Mengeot, 1978). Chez $P$. dilatatus, les hémocytes ont peut-être aussi la possibilité de libérer diverses substances pendant l'obturation de la blessure, mais il est peu probable qu'ils sécrètent le contenu de la chambre d'incubation ; en effet, ils se raréfient dès la mise en place du bourgeon, puis disparaissent pendant son développement, alors que le volume de la chambre et de son contenu augmentent. 
Il est vraisemblable que ce sont les cellules épithéliales du bourgeon qui sécrètent le contenu de la chambre et l'enveloppe du régénérat, les différenciations ultrastructurales de la membrane apicale étant certainement en rapport avec cette sécrétion. Les microvillosités observées ici sont comparables à celles qui ont été décrites par Kummel ef al. (1970) à la surface des cellules de l'épithélium des branchiostégites d'Orconectes limosus au début de la sécrétion de la cuticule. Les cellules épithéliales du bourgeon de régénération de $P$. dilatatus ne présentent ces microvillosités que par plages. Cette répartition discontinue est peut-être responsable de la disposition en réseau lâche, alvéolaire, des filaments du contenu de la chambre d'incubation. Les vésicules ouvertes au pôle apical sont probablement des vésicules d'exocytose puisque le volume de la chambre d'incubation augmente au cours de l'évolution du bourgeon. Le contenu de ces vésicules ressemble à celui des fibres épaisses situées parmi les filaments fins.

\section{Conclusion.}

Le matériel qui entoure le bourgeon de régénération répond positivement aux tests histochimiques de l'acide hyaluronique et à ceux de la chitine. Ces deux substances ont une parenté chimique : l'acide hyaluronique esł un polymère d'unités comprenant de l'acide $\mathrm{D}$-glucuronique et de la $\mathrm{N}$-acétyl-glucosamine, ef la chitine est un polymère de la $N$-acétyl-glucosamine. Sannasi (1968) a émis l'hypothèse que l'acide hyaluronique pourrait être un stade intermédiaire du métabolisme qui conduit à la synthèse de la chitine. L'exosquelette, sécrété par les cellules épithéliales du tégument intact, est riche en chitine : 34 p. 100 des substances organiques chez Mefapenaeus sp. (Dall, 1965), plus de 60 p. 100 de la cuticule complètement achevée et décalcifiée chez divers décapodes (Welinder, 1974) ; il contient également un mucopolysaccharide acide qui représente moins de 1 p. 100 du poids de la cuticule chez Hemigrapsus nudus (Meenakshi et Scheer, 1959). Cet exosquelette est sécrété pendant une période de quelques jours avant ef après l'exuviation. Le contenu de la chambre d'incubation que nous avons étudié, apparemment plus riche en MPSA et moins riche en chitine que l'exosquelette, est sécrété pendant le stade $C$ du cycle de mue, stade correspondant à un arrêt de la fonction sécrétrice des cellules épithéliales du tégument intact. Les concentrations hormonales et les ressources en métabolites pendant le stade $C$ ne sont certainement pas les mêmes que pendant l'élaboration de l'exosquelette et elles influent probablement sur la nature et la structure des productions des cellules épithéliales.

Reçu en avril 1979.

Accepté en juin 1979.

\section{Références}

ADIYODI R. G., 1972. Wound healing and regeneration in the Crab Paratelphusa hydrodromous. Int. Rev. Cytol., 32, 257-289.

BAUCHAU A. G., 1961. Régénération des péréiopodes et croissance chez les crustacés décapodes brachyoures. I. Conditions normales et rôle des pédoncules oculaires. Soc. roy. Zool. Belg., 91, 57-84. 
BAUCHAU A., DE BROUWER M. B., 1974. Etude ultrastructurale de la coagulation de l'hémolymphe chez les crustacés. J. Microsc., 19, 37-46.

BAUCHAU A. G., DE BROUWER M. B., PASSELECQ-GERIN E., MENGEOT J. C., 1975. Etude cylochimique des hémocyłes des crustacés décapodes brachyoures. Histochemistry, 45, 101-113.

BAUCHAU A. G., MENGEOT J. C., 1978. Structure et fonction des hémocytes chez les crustacés. Arch. Zool. exp. gén., 119, 227-248.

BAZIN F., DEMEUSY N., 1972. Processus de cicatrisation consécutif à l'autolomie d'un péréiopode chez le Crabe Carcinus maenas (L.). C. R. Acad. Sci. Paris, 274, 2603-2605.

BUSSON Y., 1935. Observations sur l'autotomie et la régénération de la pince chez le Bernard l'Ermite. Trav. Siat. Biol. Roscoff, 13, 133-153.

CHAPRON C., 1972. Recherches sur le rôle et le mode d'action des amines biogènes dans la régénération des oligochètes. Bull. Soc. Zool. Fr., 97, 327-331.

CHAPRON C., 1973. Etude comparée de la nature et du mode d'action des substances neurotrophiques intervenant dans la régénération des Oligochètes et des Amphibiens. $C$. R. Acad. Sci. Paris, 276, 1463-1466.

DALL W., 1965. Studies on the physiology of a shrimp. Metapenaeus sp. (Crustacea, Decapoda, Penaeidae). Austr. J. Mar. Freshw. Res., 16, 13-23.

DURLIAT M., VRANCKX R., 1976. Analyse des protéines hémocytaires d'Astacus leptodactylus. C. R. Acad. Sci. Poris, 282, 2215-2218.

GANTER P., JOLLES G., 1969 ef 1970. Histochimie normale ef pathologique (tome 1 ef 2) GauthierVillars, Paris, 1904 pp.

HOARAU F., 1973. Comportement de l'hypoderme et progression de la différenciation au cours de la régénération d'un péréiopode chez l'Isopode terrestre Helleria brevicornis Ebner. Ann. Embryol. Morph., 6, 125-135.

HUNT S., 1970. Polysaccharide-protein complexes in invertebrates. Acad. Press, 321 pp.

KUMMEL G., CLAASSEN H., KELLER R., 1970. Zur Feinstruktur von Cuticula und Epidermis beim Flusskrebs Orconectes limosus während eines Häutungszyklus. Z. Zellforsch., 109, 517-551.

LISON L., 1960. Hisfochimie ef cytochimie animales (2 vol.). Gauthier-Villars, Paris, 842 pp.

MARKS E. P., REINECKE J. P., 1964. Regenerating tissues from the cockroach leg : A system for studying in vitro. Science, 143, 961-963.

MARKS E. P., LEOPOLD R. A., 1971. Deposition of cuticular substances in vitro by leg regenerates from the cockroach, Leucophaea maderae (F.). Biol. Bull., 140, 73-83.

MEENAKSHI V. R., SCHEER B. T. (1959). Acid mucopolysaceharide of the Crustacean cuticle. Science, 130, $1189-1190$

NEEDHAM A. E., 1965. Regeneration in the arthropoda and its endocrine control, 283-323. Proc. Regeneration in Animals, North-Holl. Publ. Co., Amsterdam.

NOULIN G., MAISSIAT J., 1974. Etude du rôle de l'organe $Y$ et de l'effet de l'ecdystérone dans la régénération d'un appendice chez l'Oniscoïde Porcellio dilatatus. J. Insect. Physiol., 20, 1963 1974.

NOUVEL-Van RYSSELBERGE L., 1937. Contribution à l'étude de la mue, de la croissance ef de la régénération chez les Crustacés Natantia. Rec. Inst. Zool. Torley-Rousseau, 6, 5-161.

PEARSE A. G. E., 1968. Histochemistry. Theoretical \& Applied. Churchill, 759 pP.

SANNASI A., 1968. Hyaluronic acid in the scar tissue of cockroach. Zool. Jb. Physiol., 74, 319-327.

THIÉRY J. P., 1967. Mise en évidence des polysaccharides sur coupes fines en microscopie électronique. J. Microsc., 6, 987-1018.

THIÉRY J. P., RAMBOURG A., 1974. Cytochimie des polysaccharides. J. Microsc., 21, 225-232.

WELINDER B. S., 1974. The crustacean cuticle. I. Studies on the composition of the cuticle. Comp. Biochem. Physiol., 47 A, 779-787.

WOOD P. J., PODELWSKI J., SHENK T. E., 1971. Cytochemical observations of the hemolymph cells during coagulation in the crayfish, Orconectes virilis. J. Morph., 134, 479-488. 